\title{
Improving Urban Traffic Mobility and Reducing Emission with Day-to- day Pricing
}

\author{
Ying Liang ${ }^{1}$, Honglian $\mathrm{Li}^{2}$, Shanshan Jiang ${ }^{1, a}$, Wenyi Zhang ${ }^{1, b}$ \\ ${ }^{1}$ MOE Key Laboratory for Urban Transportation Complex Systems Theory and Technology, Beijing \\ Jiaotong University, Beijing 100044, China;
}

Beijing Vocational College of Transportation, Beijing 100096, China.

a17120819@bjtu.edu.com, bwyzhang@bjtu.edu.cn

Keywords: road pricing, day-to-day dynamics, route swapping, emission.

\begin{abstract}
The existing studies on day-to-day pricing are mainly theory-amicable, and insufficient attention is paid to practical demand on mobile efficiency and feasibility. In addition, the environment issues are valued very much in recent years in China to improve the human settlement and then support the sustainable development of a city. Seeing these, this paper constructs a day-to-day congestion pricing model to improve the mobility and environmental benefits. In this model, more practical constraints are considered, such as more practical route-swapping models, medium charging upper bound, etc. Numerical example is conducted to perform the model and algorithm. It is found that the derived pricing strategy can improve the system utility (either in mobility or in environmental benefits), and such improvement grows positively with permitted charging upper bound.
\end{abstract}

\section{Introduction}

The holding quantity of private cars in China has already exceeded one million and continues to grow rapidly in these years. The rising traffic congestion is an inescapable condition in large and growing metropolitan areas across the whole China. Furthermore, environmental pollution resulted from exhaust emissions has become increasingly severe. In order to relieve traffic congestion and reduce carbon emissions, the government has implemented a string of new regulations such as building more roads, purchase restriction, driving restriction, congestion pricing, etc. In this paper, we are interested in such an economic method, i.e., congestion pricing, which has been discusses extensively. Over all, the existing studies in congestion pricing can be divided into two classes, i.e., static pricing [1] and dynamic pricing. In reality, the transportation network is an open system, and could be interfered arbitrarily at any time. Accordingly, the network is more perhaps lying in a non-equilibrium state rather than an equilibrium state. In this condition, deploying day-to-day non-equilibrium pricing is more appropriate. As a result, this paper gives attention to day-to-day dynamic pricing, and we list below the literature review regarding this area.

Based on the marginal pricing theory, Wie and Tobin [2] proved that the dynamical day-to-day congestion pricing in a traffic network can be determined by a convex control formula. Friesz et al. [3] put forward the daily variable congestion pricing model to maximize the net present value of social welfare. Yang and Szeto [4] considered the adjustment of travelers' behavior and studied the convergence of the daily congestion pricing. After that, Yang [5] added the elastic demand of the travelers. Shortly afterwards, a novel steepest descent dynamic toll scheme is proposed by Yang et al. [6]. With the objective of minimizing the total travel cost, Tan et al. [7] studied the dynamic day-today pricing model, and took the evolution process of daily dynamic traffic flow and user heterogeneity into account. Wang et al. [8] proposed a congestion pricing model based on this to improve the flexibility of traffic network. Ye et al. [9] and Xu et al. [10] used the trial-and-error method to discuss the dynamic day-to-day pricing of traffic network. Guo et al. [11] put forward a daily congestion pricing model which was only determined by the network flow and pricing of yesterday. Liu et al. [12] explored the nonlinear distance's daily dynamic congestion pricing problem including the randomness of traffic networks. A multi-equilibrium, discrete daily dynamic congestion model was 
established, and it accepted asymmetry of the travel cost function by Han et al. [13]. More research can be found in Cheng et al. [14].

The existing studies on day-to-day congestion pricing are mainly theory-amicable, and insufficient attention is paid to practical demand on mobile efficiency and feasibility. In addition, the environment issues are valued very much in recent years in China to improve the human settlement and then support the sustainable development of a city. Seeing these, this paper constructs a day-to-day congestion pricing model to improve evolutional mobility and environmental benefits. In this model, more practical constraints are considered. Numerical example is conducted to analyze the approach. It is expected that this study can provide some beneficial theoretical reference for practical traffic managements.

\section{Mathematical Modeling}

In this section, we elaborate on the model formulation for the current problem. Two main assumptions are made in prior for this model. (1) Travel demand over the policy duration is fixed and known. (2) Travelers adjust routes according to the traffic information in previous day and follow the nonlinear min-cost-pursued swapping process [15].

To facilitate the statement and help understand the context better, we list below the symbols and notations used across the paper.

$A$ is the set of links indexed by $a \in A$.

$N$ is the time duration of the pricing policy, indexed by $n \in N$.

$W$ is the set of OD (Origin-destination) pairsindexed by $w \in W$.

$K^{w}$ is the set of effective paths between OD pair $w$, and let $k, p \in K^{w}$.

$\theta^{w}$ is a small positive constant representing travelers' reaction sensitivity.

$q^{w}$ is the travel demand between OD pair $w$.

$\delta_{a k}^{w}$ is the link-path incidence indicator, $\delta_{a k}^{w}=1$ if path $k$ uses link $a$, and $\delta_{a k}^{w}=0$ otherwise.

vot is the monetary value of time.

voe is the monetary value of emission.

$\gamma_{1}, \gamma_{2}, \gamma_{3}$ and $\gamma_{4}$ are the coefficients of $\mathrm{CO}_{2}$ emission function.

$l_{a}$ is the length of link $a$.

$t_{a}^{n}$ is the real travel time of link $a$ on day $n$.

$v_{a}^{n}$ is the flow of link $a$ on day $n$.

$f_{k}^{w n}$ is the flow of path $k$ between OD pair $w$ on day $n$.

$K_{*}^{w n}$ is the set of the shortest paths between OD pair $w$ on day $n$.

$K_{-}^{w n}$ is the supplement of the set $K_{*}^{w n}$ against the full concentration $K^{w}$.

$G_{k}^{w n}$ is the general monetary cost of path $k$ between OD pair $w$ on day $n$.

$T_{k}^{w n}$ is the travel time of path $k$ between OD pair $w$ on day $n$.

$\rho_{k p}^{w n}$ is to estimate the traffic ratio adjusting from path $k$ to path $p$ between $w$ on day $n$.

$E F_{a}^{n}$ is the emission factor of link $a$ on day $n$ with unit being $\mathrm{g} / \mathrm{km}$.

$s_{a}^{n}$ is the speed of link $a$ on day $n$, and $s_{a}^{n}=l_{a} / t_{a}^{n}$.

$x_{a}^{n}$ is the congestion pricing of link $a$ on day $n$, which is the decision variable of this model.

Given a fully connected traffic network, the mathematical model is formulated as follows.

$\min _{\mathbf{x}} Z(\mathbf{x})=\sum_{n=1}^{N} \sum_{a \in A}\left(\operatorname{vot} \cdot t_{a}^{n}(\mathbf{x})+\operatorname{voe} \cdot \frac{E F_{a}^{n}(\mathbf{x}) \cdot l_{a}}{1000}\right) \cdot v_{a}^{n}(\mathbf{x})$

$x_{a}^{n} \in\left[0, x^{+}\right], \forall a \in A, n \leq N$ 


$$
\begin{aligned}
& f_{k}^{w n}(\mathbf{x})-f_{k}^{w(n-1)}(\mathbf{x})=\left\{\begin{array}{c}
\frac{1}{\left|K_{*}^{w n}\right|} \sum_{p} f_{p}^{w(n-1)}(\mathbf{x}) \rho_{k}^{w(n-1)}(\mathbf{x}), \text { if } k \in K_{*}^{w n} \\
-f_{k}^{w(n-1)}(\mathbf{x}) \sum_{p} \rho_{k}^{w(n-1)}(\mathbf{x}), \text { if } k \in K_{-}^{w n}
\end{array}, \forall w \in W, n \in[1, N]\right. \\
& \rho_{k p}^{w n}(\mathbf{x})=1-\exp \left(-\theta\left(G_{k}^{w n}(x)-G_{p}^{w n}(x)\right)\right), \forall k \in K_{-}^{w n}, p \in K_{*}^{w n}, w \in W, n \in[1, N] \\
& G_{k}^{w n}(\mathbf{x})=v o t \cdot T_{k}^{w(n-1)}(x)+\sum_{a} \delta_{a k}^{w} x_{a}^{n}, \forall k \in K^{w}, w \in W, n \in[1, N] \\
& \sum_{k} f_{k}^{w n}(\mathbf{x})=q^{w}, \forall w \in W, n \in[0, N] ; f_{k}^{w n}(\mathbf{x}) \geq 0, \forall k \in K^{w}, w \in W, n \in[0, N] \\
& v_{a}^{n}(\mathbf{x})=\sum_{w} \sum_{k} \delta_{a k}^{w} f_{k}^{w n}(x), \forall a \in A, n \in[0, N] \\
& T_{k}^{w n}(\mathbf{x})=\sum_{a} \delta_{a k}^{w} t_{a}^{n}(x), \forall k \in K^{w}, w \in W, n \in[0, N] \\
& E F_{a}^{n}(x)=\frac{\gamma_{1}}{s_{a}^{n}(x)}+\gamma_{2} s_{a}^{n}(x)+\gamma_{3}\left(s_{a}^{n}(x)\right)^{2}+\gamma_{4}, \forall a \in A, n \in[0, N]
\end{aligned}
$$

Eq. (1) is the objective function which includes two parts: the delay cost caused by road congestion, and emission costs. Eq. (2) indicates the adjustment range of pricing for each path. Eq. (3) means the adjustment rules of the shortest path. (1) The flow on the shortest path between the same OD pair will increase, on the other hand, the flow on the non-shortest path will decrease. (2) If the shortest path between OD pairs is not unique, then the paths will be equally divided into the flow. Eq. (4) represents the ratio of the adjustment of the path $k$ between the OD pairs $w$ on the day $n$ to the amount of path $p$ traffic. Eq. (5) signifies the cost after adjusting the flow upon the effect of congestion pricing. In Eq. (6), $\sum_{k} f_{k}^{w n}(\mathbf{x})=q^{w}$ is the demand conservation constraint, and $f_{k}^{w n}(\mathbf{x}) \geq 0$ is non-negatively constrained about the path flow. Eqs. (7) and (8) are constraints regarding to intermediate variables, which states the additivity between the link and path flow, as well as the path and link travel times. In this paper, we only care for the emission of carbon dioxide. Eq. (9) is the car emission function, which is only related to speed of cars, and according to Ref. [16], $\gamma_{1}, \gamma_{2}, \gamma_{3}$ and $\gamma_{4}$ equal to $3952.113,-3.061,0.029,223.289$, respectively.

\section{Numerical Example}

The example in this section is a traffic network in real life confirmed by the instance verification and the actual utility of congestion pricing is considered as an adjustment strategy. This path starts from Beijing Jiaotong University and finally ends at the Olympic Cultural Square. Two different routes are selected and the first route is mainly along the North Second Ring Road; the second route is mainly along the North Third Ring Road. Although the second route is longer than the first, it is farther from the city center, so the road capacity is higher.

In order to simplify our observation and solution, a simplified abstraction of the background road network is illustrated in the example road network shown in Fig.1. The four numbers in each bracket respectively indicate the path label, free flow time (Unit: h), available capacity (Unit: pcu/h), and road length (Unit: km).

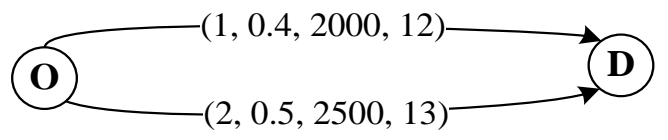

Fig. 1 A simple algorithm network

In real life, there are not intersections in these two paths. Therefore, the classical BPR function is used to calculate the travel time of each path/section each day. 


$$
c_{a}^{t}=c_{a 0}\left[1+0.15\left(\frac{v_{a}^{t}}{Y_{a}}\right)^{4}\right], \forall a \in A, n \in[0, N]
$$

where the $c_{a 0}$ and $Y_{a}$ in the formula represent the free-flow time and available capacity of the links/paths $a$, respectively (the units are omitted for ease of expression).

The model parameters are assumed as $N=30, x^{+}=\{0: 1: 4\}$ (Unit: CNY) (Note that $\{0: 1: 4\}$ denotes the set of numbers increasing from 0 to 4 by step-size 1$), \mathbf{f}^{0}=(3000,3000)^{T}($ Unit: pcu/h), $\theta=0.0226$, vot $=40($ Unit: $\mathrm{CNY} / \mathrm{h})$ and voe $=4(\mathrm{Unit}: \mathrm{CNY} / \mathrm{kg})$; and the initial step-size of algorithm is set as 1 , and the termination tolerance of iteration is set as 0.001 .

Considering the similarity between models, the compass search algorithm suggested in Ref. [17] is still used to identify the optimal solution of the current problem. The procedure for this compass search algorithm is omitted here due to limited space.

, In Fig. 2, the convergence result is shown for the current numerical example. Fig. 3 displays the flow evolution trajectory under different tolling upper bounds. In two figures, legends $\mathrm{b}, \mathrm{c}, \mathrm{d}$ and $\mathrm{e}$ point to cases $x^{+}=0, x^{+}=1, x^{+}=2, x^{+}=3, x^{+}=4$, respectively.

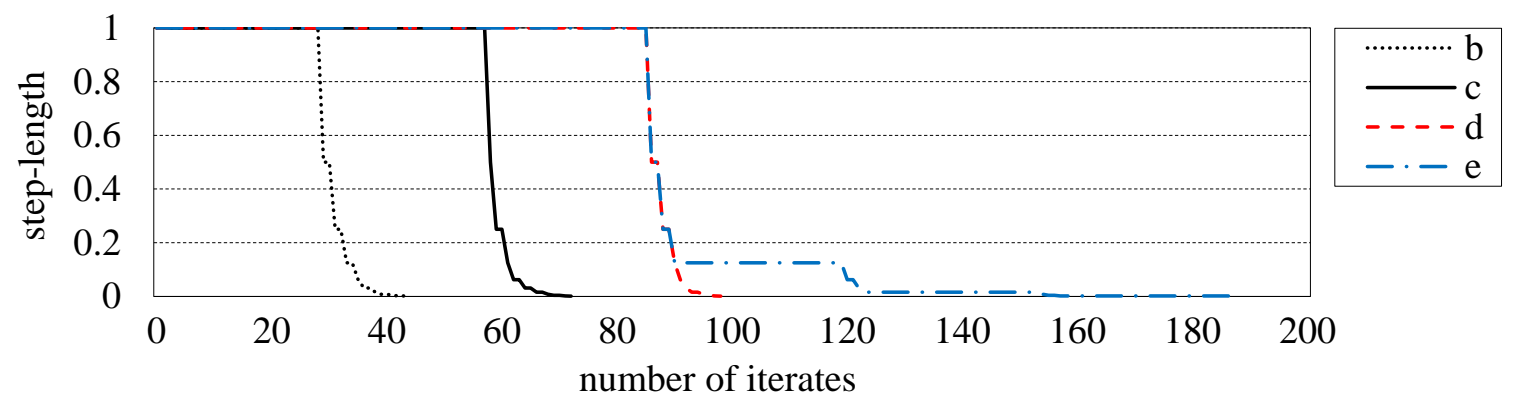

Fig. 2 The iteration process of algorithm

Fig. 2 shows that although the number of iterations corresponding to different $x^{+}$corresponding is different, the iterative steps continue to decrease until it converges; when the $x^{+}$value increases, the corresponding number of iterations will increase, which is consistent with the reality. Combining the above two points, the conclusion is that it is effective to use the improved compass search algorithm to solve the strategy adjustment model in this example. In addition, this algorithm has fewer iterations and faster convergence rate, so the algorithm is effictive to solve the current examples.

Table 1 The general objective function and its partial ones

\begin{tabular}{cccc}
\hline$x^{+}$ & Objective function $(\mathrm{CNY})$ & Delay time $(\mathrm{h})$ & Emissions $(\mathrm{kg})$ \\
\hline 0 & 8068142.54 & 117049.09 & 846544.69 \\
1 & 8045149.69 & 116640.41 & 844883.30 \\
2 & 8030693.59 & 116380.19 & 844621.49 \\
3 & 8024983.77 & 116271.99 & 843531.94 \\
4 & 8024893.25 & 116269.14 & 843526.02 \\
\hline
\end{tabular}

In Table 1, the second column is the total economic cost, the third is the time delay, and the fourth is the carbon emissions. From above, it can be seen that with $x^{+}$increasing, the total cost, time delay, and carbon emissions are all decreasing, but the reduction is gradually reduced, which is due to gradual convergence to the system optimization. When it converges to the optimization, the total cost was reduced by 43249.29 , compared with no adjustment of strategy $\left(x^{+}=0\right)$. The delay was reduced by 779.95 , and emission reduction was 3018.67 . It is proved effectively to use congestion pricing to influence the traveler's travel decisions to adjust the traffic volume of the road network. It also shows that the improved compass strategy has good search and optimization capabilities. 


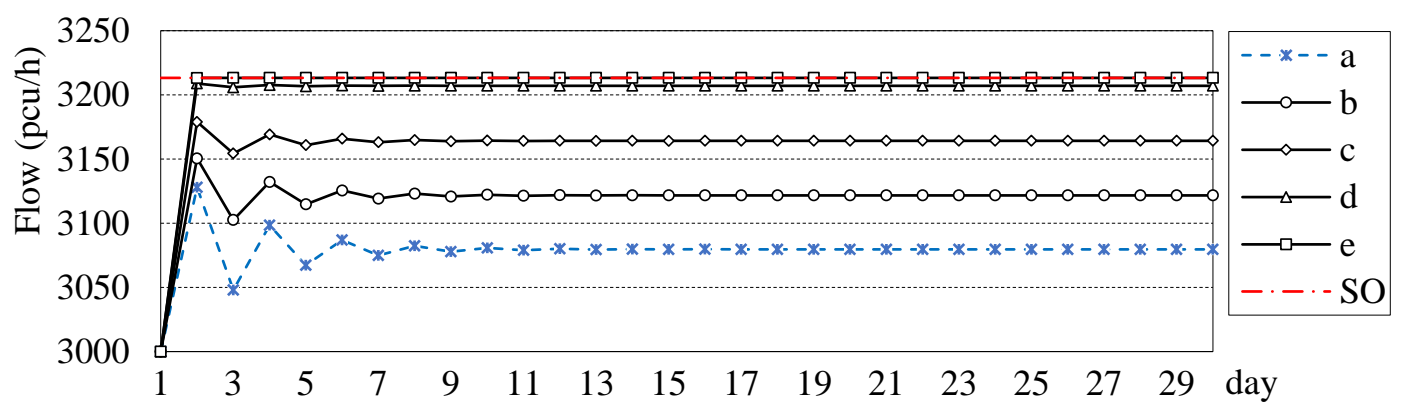

(a)

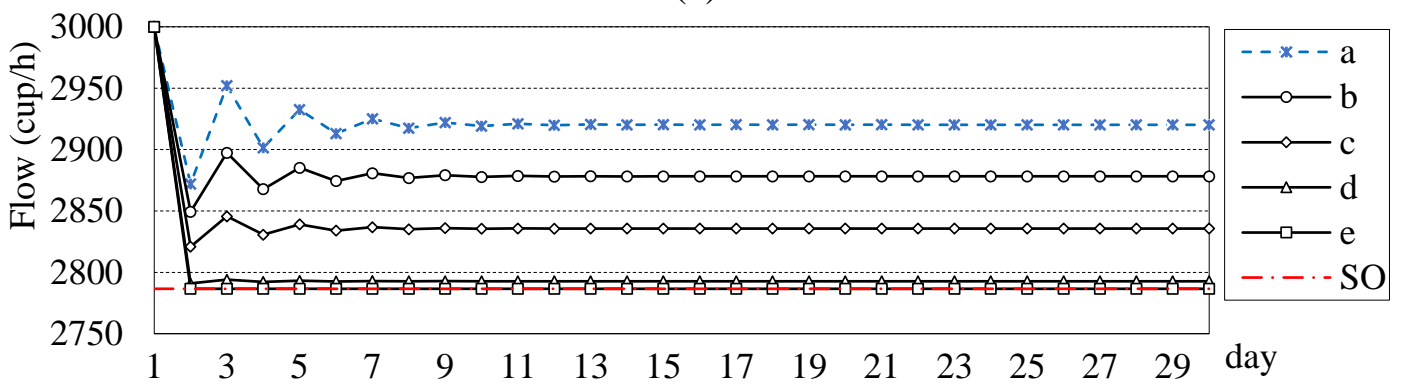

(b)

Fig. 3 Traffic evolutions under the different of $x^{+}$optimal strategies. Sub-figures a and $\mathrm{b}$ give the results of Paths 1 and 2, respectively. Legend SO gives the result under system-optimal state.

In Fig. 3, we can see that by adjusting the congestion pricing value the oscillation phenomenon is effectively reduces in the early evolution of the traffic flow. The bigger the $x^{+}$is, the better effect and the lower the required cost are. The adjustment range of the traffic adjustment is always maintained between the no strategy $\left(x^{+}=0\right)$ and the system optimization. The traffic flow gradually tends to the optimal flow value of the system with the increase of $x^{+}$in the traffic flow. It reaches the optimal state of the system when $x^{+}=4$.

\section{Summary}

This paper develops a day-to-day congestion pricing model to improve the evolutional mobility and environmental benefits for non-equilibrium traffic network. In this model, more practical constraints are considered, such as more practical route-swapping models, medium charging upper bound, etc. Numerical example is conducted to perform the model using a selected algorithm. It is found that the derived pricing strategy can effectively improve the system utility (in terms of either mobility or environmental benefits), and such improvement grows positively with the permitted charging upper bound. Moreover, for a specific network under a given travel demand, such improvements are not perpetual since the traffic evolution trajectory reaches the system optimum if the toll upper bounds increase up to a certain threshold.

\section{Acknowledgement}

This work is jointly funded by the National Natural Science Foundation of China (71601015, 91746201).

\section{References}

[1] Knight F H. Some fallacies in the interpretation of social cost[J]. Quarterly Journal of Economics, 1924, 38(4): 582-606.

[2] Wie B W, Tobin R L. Dynamic congestion pricing models for general traffic networks[J]. Transportation Research Part B, 1998, 32(5): 313-327. 
[3] Friesz T L, Bernstein D, Kydes N. Dynamic congestion pricing in disequilibrium[J]. Networks \& Spatial Economics, 2004, 4(2): 181-202.

[4] Yang F, Szeto W Y. Day-to-day dynamic congestion pricing policies towards system optimal[C]. The First International Symposium on Dynamic Traffic Assignment, 2006, 266-275.

[5] Yang F. Day-to-day dynamic optimal tolls with elastic demand[C].Transportation Research Board 87th Annual Meeting. 2008, 08-0305.

[6] Yang F, Yin Y, Lu J. Steepest descent day-to-day dynamic toll[J]. Transportation Research Record, 2008, 2039: 83-90.

[7] Tan Z, Yang H, Guo R Y. Dynamic congestion pricing with day-to-day flow evolution and user heterogeneity[J]. Transportation Research Part C, 2015, 61: 87-105.

[8] Wang Y, Liu H, Han K, et al. Day-to-day congestion pricing and network resilience[J]. Transportmetrica, 2015, 11(9): 873-895.

[9] Ye H B, Yang H, Tan Z J. Learning marginal-cost pricing via a trial-and-error procedure with day-to-day flow dynamics[J]. Transportation Research Part B, 2015, 81: 794-807.

[10] Xu M, Meng Q, Huang Z. Global convergence of the trial-and-error method for the trafficrestraint congestion-pricing scheme with day-to-day flow dynamics[J]. Transportation Research Part C, 2016, 69: $276-290$.

[11] Guo R Y, Yang H, Huang H J, et al. Day-to-day flow dynamics and congestion control[J]. Transportation Science, 2016, 50 (3): 982-997.

[12] Liu Z, Wang S, Zhou B, et al. Robust optimization of distance-based tolls in a network considering stochastic day to day dynamics[J]. Transportation Research Part C, 2017, 79: 58-72.

[13] Han L H, Wang D Z W, Hong K L, et al. Discrete-time day-to-day dynamic congestion pricing scheme considering multiple equilibria[J]. Transportation Research Part B, 2017, 104: 1-16.

[14] Cheng Q X, Liu Z Y, Liu F F, et al. Urban dynamic congestion pricing: an overview and emerging research needs[J]. International Journal of Urban Sciences, 2017, 21 (sup1): 3-18.

[15] Zhang W Y, Day-to-day traffic route-swapping model and algorithm[D], Beijing Jiaotong University, 2014.

[16] Yao E J, Song Y Y. Study on eco-route planning algorithm and environmental impact assessment[J]. Journal of Intelligent Transportation Systems, 2013, 17(1): 42-53.

[17] Zhang W Y, Zhang H M, Guan W, et al. Managing day-to-day network traffic evolution via an altering ex-post information release strategy[J]. Journal of Transportation Engineering, Part A: System, 2018, 144(7): 04018208. 